\title{
Rate-control vs rhythm-control of atrial fibrillation in elderly patients. From new, age-oriented outcomes to a more complex management strategy
}

\author{
Stefano Fumagalli, Serena Boni, Simone Pupo, Marta Migliorini, Irene Marozzi, \\ Eleonora Barghini, Flavia Sacco, Niccolò Marchionni
}

\author{
Geriatric Intensive Care Unit and Geriatric Arrhythmia Unit, University of Florence and AOU Careggi, \\ Florence, Italy
}

\begin{abstract}
Atrial fibrillation (AF) is the most frequent arrhythmia in elderly people. Findings derived from clinical trials seem to demonstrate that a rate-control strategy of $\mathrm{AF}$ in aged patients improves prognosis if compared to a rhythm-control one. However, epidemiological studies concordantly show that the arrhythmia is associated to increased hospitalization and mortality rates. In last years, the proportion of patients admitted to hospital for AF has progressively increased; this trend is observed in subjects $\geq 75$ and $\geq 85$ years, while no change was found in younger cohorts. Importantly, in aged individuals, probably because of the loss of atrial activity, the increase of heart rate and the irregularity of RR intervals, AF begins a vicious cycle, leading from heart failure, through the compromise of functional and neurocognitive status, to overt disability, dementia and increased mortality. Evidence specifically aimed at clarifying the effects of arrhythmia management on outcomes characteristic of aged people is completely lacking. In the elderly, the question regarding the effects of a rate- or a rhythm-control strategy of AF should be considered as an aspect of a more complex strategy, addressed to reduce disability and hospitalizations, and to improve quality of life and survival.
\end{abstract}

Corresponding author: Stefano Fumagalli, Geriatric Intensive Care Unit and Geriatric Arrhythmia Unit, University of Florence and AOU Careggi, Viale Pieraccini 6, 50139 Florence, Italy. Tel. +39.055.2758135.

E-mail: stefano.fumagalli@unifi.it

Key words: Atrial fibrillation; elderly; prognosis; rate-control; rhythm-control.

Received for publication: 24 April 2018

Accepted for publication: 11 May 2018

(C) Copyright S. Fumagalli et al., 2018

Tipografia PI-ME Editrice, Italy

Monaldi Archives for Chest Disease 2018; 88:955

doi: 10.4081/monaldi.2018.955

This article is distributed under the terms of the Creative Commons Attribution Noncommercial License (by-nc 4.0) which permits any noncommercial use, distribution, and reproduction in any medium, provided the original author(s) and source are credited.

\section{Atrial fibrillation, hospitalizations and mortality in elderly subjects}

Atrial fibrillation (AF) is the most frequent arrhythmia of elderly people with a prevalence of $23 \%$ at ages $\geq 85$ years [1]. Real world data show that, in old subjects, a rate-control approach to AF is preferred over a rhythm-control one [2]. Indeed, the results of a sub-analysis of the AFFIRM (Atrial Fibrillation Follow-up Investigation of Rhythm Management) Trial demonstrated that in the group of patients aged 70-80 years, after 3.4 years of follow-up, all-cause mortality was significantly higher in the rhythm-control arm than in the rate-control one (23 vs 18\%, $\mathrm{p}=0.010$ ) [3]. However, some evidence seems to demonstrate that the arrhythmia has a not completely favorable and benign course. In particular, the same AFFIRM Investigators showed that, when compared to the presence of AF, the finding of sinus rhythm at the EKG was an independent predictor of higher survival at the end of the follow-up [4]. The results of the Olmsted County Study evidenced in 4618 subjects with a mean age of 73 years that the hazard ratio (HR) of mortality was 9.62 times higher than that expected in the general population after the first 4 months from the diagnosis of AF. Interestingly, mortality risk remained higher $(\mathrm{HR}=1.66)$ even after that period and until the end of the 5.3 years of follow-up [5]. More recently, an analysis from the 0lmsted County Study, demonstrated that, when compared to controls, not only mortality was higher in AF subjects living in community (mean age: $74 \pm 14$ years; 10.5 vs 4.7 events per 100 person-years, $\mathrm{p}<0.001$ ), but also the cumulative rate of hospitalizations ( $58.8 \mathrm{vs} 26.4$ events per 100 person-years, $\mathrm{p}<0.05$ ) [6]. Indeed, administrative data collected among the Medicare fee-for-service beneficiaries $(\mathrm{N}=68.374 .904)$ showed that hospitalization rates for AF progressively and continuously grew from 1999 to 2013 in subjects aged $\geq 85$ years $(2013-\mathrm{N}=1261$ per 100.000 person-years; $+2.22 \%$ per year) and $75-84$ years $(2013-\mathrm{N}=946$ per 100.000 person-years; $+0.99 \%$ per year), but not in those aged 65-74 years [7].

\section{AF from a geriatric point of view}

Older population with AF often presents a high burden of comorbid conditions; if compared to a matched cohort of subjects without the arrhythmia, it was possible to find not only an increased prevalence of chronic heart failure, coronary artery disease and hypertension, but also a higher proportion of subjects with non-cardiovascular conditions, such as cancer, chronic obstructive pulmonary disease, chronic renal failure, diabetes and obesity [6]. On the whole, the about 2.5 millions of Medicare beneficiaries with AF have 6 associated comorbidities. Aspiration pneumonia and urinary tract infections are among the 
ten most frequent main diseases in elderly hospitalized patients with $\mathrm{AF}$ as a secondary diagnosis [8]. In this sense, if the arrhythmia develops in elderly population, it may be considered as a marker of a complex clinical condition and, perhaps, of frailty [9]. Indeed, the term "disability-adjusted life-years" was recently coined when speaking of the complications related to AF. In particular, the impact of the arrhythmia on functional profile starts to increase in an age-related manner after 60 years of age both in men and women. This important link can be explained by some considerations. First of all, the aging process, cardiovascular risk factors and metabolic alterations can all predispose to AF development. When the arrhythmia initiates, heart rate usually increases while atrial contraction and atrio-ventricular synchrony are lost. This changes usually associates with a reduction of myocardial contractility and of cardiac output. The events we described can be related to stroke or to heart failure development [10]. The synergistic interaction between $\mathrm{AF}$ and heart failure can explain the worse prognosis also in patients implanted with a device for cardiac resynchronization therapy. At this regard, the presence of a permanent AF, when compared to what observed when sinus rhythm was recorded, associated to a significantly higher mortality risk $(\mathrm{HR}=1.63,95 \% \mathrm{CI}=1$ $16-2.30, \mathrm{p}=0.005)$ in the 1787 patients of the InSync/InSync ICD Italian Registry [11]. Importantly, the Cardiovascular Health Study Investigators showed that neuro-cognitive profile of subjects experiencing a new AF episode started to diverge, becoming worse than that observed in the remaining part of the enrolled population. This experience, led in 5150 patients followed-up for 7 years, represents the first demonstration in a study with a longitudinal design of the tight link between the arrhythmia and dementia [12]. Furthermore, it had been already shown that disturbances of learning and memory significantly grow following $\mathrm{AF}$ burden, reaching the highest alteration when chronic forms of the arrhythmia are present [13]. Similarly, in the Age, Gene/Environment Susceptibility-Reykjavik Study, AF population had a lower score in memory tests than control individuals. Importantly, brain volume, evaluated with magnetic resonance imaging, progressively reduced going from sinus rhythm to paroxysmal and persistent / permanent AF subjects [14]. More recently, the Health, Aging, and Body Composition Study, in a prospective cohort of community-dwelling older adults $(\mathrm{N}=2753)$, demonstrated that physical performance, evaluated with a specific battery of tests (grip strength, 2-minute walk distance, 400-m walking time), had a greater decline after an incident AF than that observed with a persistent sinus rhythm. This association was verified in people aged 70, 74, 78 and 82 years during a follow-up lasting 10 years [10]. In summary, in the arrhythmic population, the increased prevalence of cerebrovascular disease, neuro-cognitive disorders and heart failure, and the higher burden of comorbidities could exert a great influence on physical performance and functional status, enforcing a path leading from disturbances of mobility, through frank disability and dementia, to higher mortality rates [10].

\section{Rate- or rhythm-control in elderly AF patients}

Evidence regarding the choice between a rate- or a rhythm-control strategy in elderly AF patients is lacking. In a recent scientific statement about knowledge gaps in cardiovascular care of the older adult population by the American Heart Association, the American College of Cardiology and the American Geriatrics Society, when speaking of AF and atrial flutter, it is reported that even if most trials have enrolled patients without an upper age limit, age of study cohorts is 5-10 years younger than the average age of patients with AF in the "real world". Therefore, it is unknown whether the findings of these studies can be generalized to patients $\geq 75$ years of age, and especially those $\geq 85$ years of age [15]. In the same text, it is explicitly specified and recommended that studies are needed to observe if differences exist between rate- and rhythm-control strategies on clinical outcomes relevant to older subjects, such as quality of life and functional status [15]. At this regard, we conducted a small, preliminary study, aimed at evaluating the impact of sinus rhythm restoration on physical performance of aged patients with persistent AF.

For this purpose, we studied all consecutive subjects undergoing external cardioversion (ECV) of the arrhythmia in a day-hospital setting. At baseline and at the follow-up, patients were assessed using the Short Physical Performance Battery (SPPB). More in detail, SPPB is a tool of the Comprehensive Geriatric Assessment, exploring functional status, evaluating balance, gait speed, strength and endurance. The test scores from 0 to 12 (lowest to highest performance), and it is able to predict incident disability and mortality. We enrolled 46 patients (age: $77 \pm 7$ years). At baseline, the median value of SPPB was 10/12 and it was inversely associated with the $\mathrm{CHA}_{2} \mathrm{DS}_{2}$-VASc score and the presence of depressive symptoms measured with the Geriatric Depression Scale. After about 4 months, sinus rhythm was observed in 24/46 patients (52.2\%), and it was associated with a significant improvement of physical functioning, which was absent in patients with AF relapse (Figure 1). The effects linked to the presence of sinus rhythm persisted also at multivariate analysis, with a SPPB score $1.1 \pm 0.44$ points higher $(p=0.018)$ than that observed when a new arrhythmic event occurred. These are preliminary data obtained in a small population. If they will be confirmed in larger series of patients, the debate on which strategy adopt in elderly AF individuals will be enriched by new endpoints, more pertinent to aged subjects.

\section{Conclusions}

$\mathrm{AF}$ is the most frequent arrhythmia of elderly people. It associates with a more complex clinical condition. AF seems to be responsible in community-dwelling individuals of higher hospitalization rates, increased mortality and dementia and disability development. Evidence guiding the management of older patients is presently lacking. Until the results of new and specifically age-oriented studies will be completed, when treating older arrhythmic subjects, we should not just focus on the alternative between rate- and rhythm control strategies, but aim to preserve functional and cognitive status, and to reduce the weight of comorbid conditions, hospitalization rates and mortality.

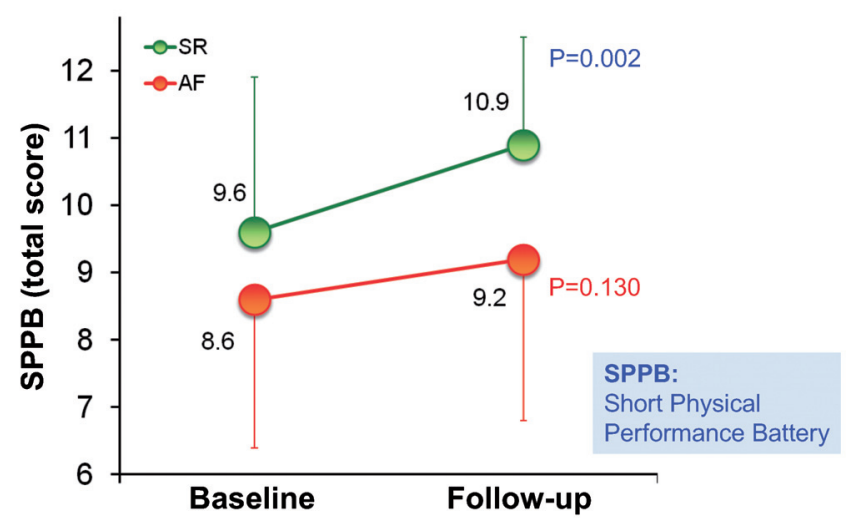

Figure 1. Changes of Short Physical Performance Battery total score after external cardioversion of atrial fibrillation, by rhythm at follow-up. SR, sinus rhythm; AF, atrial fibrillation. 


\section{References}

1. Miyasaka Y, Barnes ME, Gersh BJ, et al. Secular trends in incidence of atrial fibrillation in Olmsted County, Minnesota, 1980 to 2000 , and implications on the projections for future prevalence. Circulation 2006;114:119-25.

2. Fumagalli S, Said SAM, Laroche $\mathrm{C}$, et al. Age-related differences in presentation, treatment, and outcome of patients with atrial fibrillation in Europe. The EORP-AF General Pilot Registry (EURObservational Research Programme-Atrial Fibrillation). JACC Clinical Electrophysiol 2015;1:326-34.

3. Shariff N, Desai RV, Patel K, et al. Rate-control versus rhythmcontrol strategies and outcomes in septuagenarians with atrial fibrillation. Am J Med 2013;126:887-93.

4. Corley SD, Epstein AE, DiMarco JP, et al. Relationships between sinus rhythm, treatment, and survival in the Atrial Fibrillation Follow-Up Investigation of Rhythm Management (AFFIRM) Study. Circulation 2004;109:1509-13.

5. Miyasaka Y, Barnes ME, Bailey KR, et al. Mortality trends in patients diagnosed with first atrial fibrillation: a 21-year community-based study. J Am Coll Cardiol 2007;49:986-92.

6. Chamberlain AM, Alonso A, Gersh BJ, et al. Multimorbidity and the risk of hospitalization and death in atrial fibrillation: A populationbased study. Am Heart J 2017;185:74-84.

7. Freeman JV, Wang Y, Akar J, et al. National trends in atrial fibrillation hospitalization, readmission, and mortality for medicare beneficiaries, 1999-2013. Circulation 2017;135:1227-39.
8. Coyne KS, Paramore C, Grandy S, et al. Assessing the direct costs of treating nonvalvular atrial fibrillation in the United States. Value Health 2006;9:348-56.

9. Fumagalli S, Tarantini F, Guarducci L, et al. Atrial fibrillation is a possible marker of frailty in hospitalized patients: results of the GIFA Study. Aging Clin Exp Res 2010;22:129-33.

10. Magnani JW, Wang N, Benjamin EJ, et al. Atrial Fibrillation and declining physical performance in older adults: The health, aging, and body composition study. Circ Arrhythm Electrophysiol 2016;9:e003525.

11. Fumagalli S, Valsecchi S, Boriani G, et al. Comparison of the usefulness of cardiac resynchronization therapy in three agegroups ( $<65,65-74$ and $>/=75$ Years) (from the InSync/InSync ICD Italian Registry). Am J Cardiol 2011;107:1510-6.

12. Thacker EL, McKnight B, Psaty BM, et al. Atrial fibrillation and cognitive decline: a longitudinal cohort study. Neurology 2013;81:119-25.

13. Knecht S, Oelschlager C, Duning $\mathrm{T}$, et al. Atrial fibrillation in stroke-free patients is associated with memory impairment and hippocampal atrophy. Eur Heart J 2008;29:2125-32.

14. Stefansdottir H, Arnar DO, Aspelund T, et al. Atrial fibrillation is associated with reduced brain volume and cognitive function independent of cerebral infarcts. Stroke 2013;44:1020-5.

15. Rich MW, Chyun DA, Skolnick AH, et al. Knowledge gaps in cardiovascular care of the older adult population: A scientific statement from the American Heart Association, American College of Cardiology, and American Geriatrics Society. J Am Coll Cardiol 2016;67:2419-40. 